University of Nebraska - Lincoln

DigitalCommons@University of Nebraska - Lincoln

John R. Hardy Papers

Research Papers in Physics and Astronomy

8-15-1972

\title{
Frenkel Defects in Alkali Halides
}

\author{
Paul Schulze \\ University of Nebraska - Lincoln \\ John Hardy \\ University of Nebraska - Lincoln
}

Follow this and additional works at: https://digitalcommons.unl.edu/physicshardy

Part of the Physics Commons

Schulze, Paul and Hardy, John, "Frenkel Defects in Alkali Halides" (1972). John R. Hardy Papers. 20. https://digitalcommons.unl.edu/physicshardy/20

This Article is brought to you for free and open access by the Research Papers in Physics and Astronomy at DigitalCommons@University of Nebraska - Lincoln. It has been accepted for inclusion in John R. Hardy Papers by an authorized administrator of DigitalCommons@University of Nebraska - Lincoln. 


\section{Frenkel Defects in Alkali Halides ${ }^{\dagger}$}

Paul D. Schulze* and John R. Hardy

Behlen Laboratory of Physics, University of Nebraska, Lincoln, Nebraska 68508

(Received 31 January 1972)

The method of lattice statics and the deformation dipole model have been applied to Frenkel defects in ionic crystals. For isolated interstitials, all short-ranged repulsive and Coulombdefect host-lattice ion forces were evaluated at the relaxed configuration. The zero-order Coulomb force was allowed to act on the remainder of the host-lattice ions past first neighbors. We have calculated Frenkel-pair formation energies, ionic displacements, and ionic polarizations in the twelve $\mathrm{Na}, \mathrm{K}, \mathrm{Rb}$ halides. Our results show over all that, in ionic crystals of the rocksalt structure, Schottky defect pairs are more likely to be present than Frenkel defect pairs.

\section{INTRODUCTION}

In an earlier paper, ${ }^{1}$ we applied the method of lattice statics and the deformation dipole model to Schottky defects in ionic crystals. In this paper we shall consider the presentation of Ref. 1 as it applies to Frenkel defects in ionic crystals.

The Frenkel-pair formation energy is defined as the energy necessary to move an ion, anion, or cation, from a normal lattice site into a well-removed interstitial site. The interstitial position considered here is located in the tetrahedral void formed by four anions and four cations. The formation energy can be broken into two parts: (i) the energy needed to remove the ion from the interior of the lattice to infinity, and (ii) the energy needed to bring the ion from infinity and place it at an interstitial position. The first contribution has already been calculated in Ref. 1 .

In this work we have determined the Frenkel formation energy, for both anion and cation interstitials, by allowing all nearest-neighbor defect host- 
lattice ion interactions to be evaluated at the relaxed-lattice configuration, and by considering defect interactions with farther neighbors in zero order with respect to the forces. This approach is chosen rather than expanding the energy for nearest neighbors (NN) as for vacancies since the interactions herein are additional energy contributions to the total energy.

\section{SHORT-RANGE INTERACTIONS}

The Huggins-Mayer short-range repulsive-potential-energy form including the attractive van der Waals potential was used for the interaction of the interstitial with its eight NN.

The energy necessary to move an ion from infinity into the interstitial position is just equal to the work done against the short-range forces since at this position the Madelung energy is zero. This follows easily from an application of the Evjen $\operatorname{method}^{2}$ or by direct summation, i. e., the neutral concentric shells about the interstitial position each give a zero net contribution to the Madelung energy. In Table I, the calculated values for the interstitial overlap energy are given for both cations and anions using the potential-energy function

$$
W_{I}^{ \pm}=4 \phi_{\mathrm{N} \mathrm{N}}( \pm \pm)+4 \phi_{\mathrm{NN}}(+-),
$$

where $\phi_{\mathrm{NN}}$ includes both the Huggins-Mayer repulsive and van der Waals attractive interactions. Specifically,

$$
\phi_{\mathrm{NN}}(i j)=-\frac{c_{i j}}{r_{0}^{\prime 6}}-\frac{d_{i j}}{r_{0}^{\prime 8}}+\lambda_{i j}^{\prime} e^{-r_{0}^{\prime} / \rho_{i j}^{\prime}},
$$

where

$$
\lambda_{i j}^{\prime}=b_{i j}\left(1+z_{i} / n_{i}+z_{j} / n_{j}\right) e^{\left(r_{i}+r_{j}\right) / \rho_{i j}^{\prime}}
$$

and $r^{\prime}=\frac{1}{2} \sqrt{3} r_{0}$. Here $r_{i}, z_{i}, n_{i}, b_{i j}$, and $\rho^{\prime}$ are, respectively, the ionic radius, the net ionic charge, the number of outer electrons, a constant characterizing the overlap between ions, and a screening parameter. The $c_{i j}$ and $d_{i j}$ are the dipole-dipole and dipole-quadrupole van der Waals coefficients calculated by Mayer, ${ }^{3}$ and the parameters $\lambda^{\prime}$ and $\rho^{\prime}$ were taken from Fumi and Tosi. ${ }^{4}$

In Ref. 1 the importance of the contribution that the missing deformation dipoles played in the determination of the Schottky-pair formation energy was shown. Similarly, for the interstitial problem one could consider additional deformation dipoles generated on the four NN anions about the interstitial ion. However, since at present it is not known how to adequately describe anion-anion deformation, this short-range interaction has not been included for either type of interstitial ion. Within this limitation, the short-range interactions can be satisfactorily described by the potentialenergy forms given in Eq. (1).

\section{FOURIER- TRANSFORMED FORCES AND FIELDS}

The Fourier transform of any force over the eight $\mathrm{NN}$ to a cation interstitial can be written

$$
\left(\begin{array}{l}
G_{\alpha}^{1}(\overrightarrow{\mathrm{q}}) \\
G_{\alpha}^{2}(\overrightarrow{\mathrm{q}})
\end{array}\right)=\frac{4 \sqrt{3}}{3}\left(\begin{array}{l}
\left(\cos \frac{1}{2} \pi q_{\alpha} \sin \frac{1}{2} \pi q_{\beta} \sin \frac{1}{2} \pi q_{\gamma}-i \sin \frac{1}{2} \pi q_{\alpha} \cos \frac{1}{2} \pi q_{\beta} \cos \frac{1}{2} \pi q_{\gamma}\right) V_{R}^{1} \\
\left(-\cos \frac{1}{2} \pi q_{\alpha} \sin \frac{1}{2} \pi q_{\beta} \sin \frac{1}{2} \pi q_{\gamma}-i \sin \frac{1}{2} \pi q_{\alpha} \cos \frac{1}{2} \pi q_{\beta} \cos \frac{1}{2} \pi q_{\gamma}\right) V_{R}^{2}
\end{array}\right),
$$

where $V_{R}^{K}$ is to be taken as the magnitude of the radial force on the $\kappa$ th-type ion about the interstitial in direct space, and $\alpha, \beta=1,2,3$ are the Cartesian components. This force is

$$
V_{R}^{\kappa}=-\left(\frac{\partial \phi(r)}{\partial r}\right)_{\left|\overrightarrow{\mathrm{r}}^{\lambda}+\vec{\xi}^{\lambda}\right|}
$$

These Fourier-transformed forces are complex as is the case when the defect is not at a site of inversion symmetry.

Since all defect NN forces are to be evaluated at the relaxed lattice configuration, the Fouriertransformed defect electric field $E_{\alpha, 0}(\vec{q})$, must be modified since it contains the zero-order field contribution from nearest neighbors. The easiest possible way in which to do this to modify $E_{\alpha, 0}(\vec{q})$ indirectly through the use of Eq. (2). Thus, utilizing the fact that Fourier transforms are distributive, and setting $V_{R}^{K}=1$ in Eq. (2), we write

$$
E_{\alpha}^{\prime}(\overrightarrow{\mathrm{q}})=E_{\alpha, 0}(\overrightarrow{\mathrm{q}})+\Delta E^{\kappa} G_{\alpha}^{k}(\overrightarrow{\mathrm{q}}),
$$

where

$$
\Delta E^{\kappa}=e_{D}\left[\left(r_{0}^{\prime}+\xi_{R}^{\kappa}\right)^{-2}-\left(r_{0}^{\prime}\right)^{-2}\right] .
$$

Here $\xi_{R}^{k}$ is the radial displacement of the $\kappa$ th nearest neighbor, and $e_{D}$ is the defect charge.

In considering Schottky defects, Ref. 1, the potential energy of an imperfect lattice was expanded to terms quadratic in the displacements. Thus, for interstitial neighbors beyond the second shell, we have used the zero-order-approximation expressions of Ref. 1. Since ions in the first shell are to be allowed to relax, we minimize the potential energy for the imperfect lattice,

$$
W=W_{0}+\sum_{\lambda(N N)} \psi\left(\left|\vec{r}^{\lambda}+\vec{\xi}^{\lambda}\right|\right)+\frac{1}{2} \sum_{\substack{\alpha, \beta \\ \lambda ; \lambda^{\prime}}} \xi_{\alpha}^{\lambda} \phi_{\alpha \beta}^{\lambda \lambda^{\prime}} \xi_{\beta}^{\lambda^{\prime}},
$$

where $\lambda$ is a contraction for the $l$ th unit cell and $\kappa$ th ion, with respect to the ionic displacements without expanding the pairwise potential energy between the interstitial ion and its $\lambda$ th NN. Thus we can find the dipole moments, ionic displacements, and 
TABLE I. Calculated interstitial overlap energy $W_{I}$, calculated energies to remove a cation and an anion to infinity, $E$, in approximation II; energy to bring ion from infinity to interstitial position, $E_{I}$; and Frenkel-pair formation energy $E_{F}$ for cations (+) and anions (-). All energies are in $\mathrm{eV}$.

\begin{tabular}{llllllllllllll}
\hline \hline & $\mathrm{NaF}$ & $\mathrm{NaCl}$ & $\mathrm{NaBr}$ & $\mathrm{NaI}$ & $\mathrm{KF}$ & $\mathrm{KCl}$ & $\mathrm{KBr}$ & $\mathrm{KI}$ & $\mathrm{RbF}$ & $\mathrm{RbCl}$ & $\mathrm{RbBr}$ & $\mathrm{RbI}$ \\
\hline$W_{I}^{+}$ & 5.646 & 3.011 & 2.593 & 2.147 & 9.037 & 3.995 & 3.284 & 2.579 & 12.001 & 5.010 & 3.921 & 2.883 \\
$W_{I}$ & 2.884 & 4.934 & 6.304 & 9.526 & 2.104 & 2.966 & 3.808 & 5.138 & 1.937 & 2.565 & 2.956 & 3.914 \\
$E_{+}(\mathrm{NNN})^{\mathrm{a}}$ & 5.972 & 5.152 & 4.859 & 4.322 & 4.996 & 4.720 & 4.518 & 4.193 & 4.679 & 4.498 & 4.341 & 4.071 \\
$E_{-}(\mathrm{NNN})^{\mathrm{a}}$ & 5.967 & 5.087 & 4.865 & 4.586 & 5.317 & 4.772 & 4.613 & 4.420 & 5.011 & 4.601 & 4.465 & 4.295 \\
$E_{I}^{+}$ & -2.444 & -2.271 & -2.301 & -2.317 & -0.723 & -1.259 & -1.360 & -1.460 & 0.025 & -0.786 & -0.987 & -1.372 \\
$E_{I}^{-}$ & -2.572 & -0.485 & -0.028 & 0.559 & -2.746 & -1.038 & -0.440 & -0.161 & -2.660 & -1.086 & -0.788 & -0.402 \\
$E_{F}^{+}$ & 3.528 & 2.881 & 2.558 & 2.005 & 4.273 & 3.461 & 3.158 & 2.733 & 4.704 & 3.712 & 3.354 & 2.699 \\
$E_{F}^{-}$ & 3.395 & 4.602 & 4.837 & 5.145 & 2.571 & 3.734 & 4.173 & 4.259 & 2.351 & 3.515 & 3.677 & 3.893 \\
\hline \hline
\end{tabular}

${ }^{2}$ Paul D. Schulze and John R. Hardy, Phys。Rev. B $\underline{5}, 3270$ (1972).

defect-formation energy following the same procedures discussed in Ref. 1.

\section{FRENKEL-DEFECT FORMATION ENERGY}

The Fourier-transformed total dipole moment, ionic displacements, and defect-formation energy, respectively, can thus be written in matrix form

$$
\begin{aligned}
& \mu=C^{-1} \alpha\left[E^{\prime}+U H(1+U \tilde{S}) \xi\right]+\tilde{S} \xi, \\
& \xi=M^{-1}\left[V+\tilde{F} \mu+\left(U^{-1}+S\right) \tilde{C}^{-1} E^{\prime}\right],
\end{aligned}
$$

and

$$
\begin{aligned}
E_{I}=W_{I}+\left[\Phi^{R}-\Phi_{0}^{R}\right] & +U^{-1}\left[\Phi^{C}-\Phi_{0}^{C}\right]-\frac{1}{2} \tilde{E}^{\prime} C^{-1} \alpha E^{\prime} \\
& -\frac{1}{2} \tilde{E}^{\prime} \mu+\frac{1}{2} \tilde{E}^{\prime} U^{-1} \xi+\frac{1}{2} \tilde{V} \xi+\frac{1}{2} \tilde{\mu} F \xi,
\end{aligned}
$$

where we have the matrix elements

$$
V \equiv V_{\alpha}^{\kappa}\left(\left|\overrightarrow{\mathbf{r}}^{\lambda}+\vec{\xi}^{\lambda}\right|\right)=-\left(\frac{\partial \psi^{\kappa}(r)}{\partial x_{\alpha}}\right)_{\left|\overrightarrow{\mathbf{r}}^{\lambda}+\vec{\xi}^{\lambda}\right|}
$$

for the short-range overlap interactions, and

$$
F \equiv F_{\alpha \beta}^{\kappa}\left(\left|\overrightarrow{\mathrm{r}}^{\lambda}+\vec{\xi}^{\lambda}\right|\right)=\left(\frac{\partial E_{\beta}^{\kappa}(r)}{\partial x_{\alpha}}\right)_{\mid \overrightarrow{\mathrm{r}}^{\lambda}+\vec{\xi}^{\lambda_{1}}}
$$

for the Coulomb field gradient. All other matrix elements have been defined in Ref. 1 .

\section{v. CALCULATIONS}

Since the forces and fields on NN are evaluated at the relaxed-lattice positions, it is necessary to iterate in order to obtain the displacements as well as the field-gradient contributions. The forceconstant matrix $M$ in Eq. (3) is the same one as was used in approximation II for the Schottky defects obtained in Ref. 1 and includes next-nearestneighbor (NNN) anion-anion interactions as well as the noncentral angle-bending force.

The actual calculations were carried out on an IBM
$360 / 65$ computer where again we have considered 8000 distinct wave vectors $\vec{q}$ in the first Brillouin zone and have extrapolated the relaxation energies to the case for which $N=\infty$ by the procedure used in Ref. 1. The extrapolated values for the energy necessary to place a cation in the interstitial site from infinity $E_{I}^{+}$and the corresponding energy for the anion $E_{I}^{-}$are given in Table I for each of the twelve alkali halides considered in this work. In this table are also given the cation and anion Frenkel-pair formation energies which are defined by the equation $E_{F}^{+}=E_{I}^{+}+E_{ \pm}^{v}$, where $E_{ \pm}^{v}$ is the energy necessary to remove an ion from a perfect lattice to infinity. In order to be consistent with the defect host-lattice ion interactions considered for interstitial defects, the value for $E_{ \pm}^{v}$ was taken from Ref. 1 for vacancies in approximation II for the NNN model, i. e., $E_{ \pm}^{v}=E_{ \pm}(\mathrm{NNN})$ and are listed for convenience in Table I. In other words, the same force-constant matrix $M$ and the same defect interactions are used. All other input data used for the calculation of Frenkel pair formation energies were identical to those used in Ref. 1.

The displacements and total dipole moments for the neighbors of interstitials in $\mathrm{NaCl}$ are given in Table II for both cation and anion interstitials for 8000 wave vectors. ${ }^{5}$

\section{SUMMARY AND DISCUSSION}

The Frenkel-pair formation energies in alkali halides have been calculated by the method of lattice statics using the deformation dipole model for polarizable ionic crystals. The perfect-lattice forceconstant matrix contained second-neighbor anionanion short-range interactions and a noncentral angle-bending force. The defect, located at the body-centered interstitial position, was allowed to 
TABLE II. Components of displacement $\vec{\xi}$ in percentage of $r_{0}$, and total dipole moment $\vec{\mu}$ in percentage of $e r_{0}$ in NaCl for cation $(+)$ and anion(-) interstitials. Components directed outward from the interstitial have the same sign as $2 l_{\alpha}$.

\begin{tabular}{|c|c|c|c|c|c|c|c|c|c|c|c|c|}
\hline \multirow{2}{*}{$\begin{array}{l}\text { Neighbor } \\
2\left(l_{1}, l_{2}, l_{3}\right)\end{array}$} & \multicolumn{3}{|c|}{$(+)$} & \multicolumn{3}{|c|}{$(-)$} & \multicolumn{3}{|c|}{$(+)$} & \multicolumn{3}{|c|}{$(-)$} \\
\hline & $\xi_{1}$ & $\xi_{2}$ & $\xi_{3}$ & $\xi_{1}$ & $\xi_{2}$ & $\xi_{3}$ & $\mu_{1}$ & $\mu_{2}$ & $\mu_{3}$ & $\mu_{1}$ & $\mu_{2}$ & $\mu_{3}$ \\
\hline $1 \overline{1} 1$ & 8.928 & -8.928 & 8.928 & -1.157 & 1.157 & -1.157 & 0.117 & -0.117 & 0.117 & -0.357 & 0.357 & -0.357 \\
\hline $\begin{array}{lll}311 & 1\end{array}$ & 2.989 & -0.596 & -0.596 & 0.504 & 0.795 & 0.795 & 0.266 & 0.023 & 0.023 & -0.375 & 0.013 & 0.013 \\
\hline $33 \frac{1}{1}$ & 2.328 & 2.328 & -0.614 & -0.491 & -0.491 & 0.592 & 0.081 & 0.081 & -0.013 & -0.051 & -0.051 & 0.019 \\
\hline 333 & 0.030 & 0.030 & 0.030 & -0.138 & -0.138 & -0.138 & 0.028 & 0.028 & 0.028 & -0.027 & -0.027 & -0.027 \\
\hline 351 & 0.238 & 0.530 & 0.028 & 0.352 & 0.355 & 0.086 & 0.027 & 0.078 & 0.011 & -0.011 & -0.091 & -0.005 \\
\hline $51 \overline{1}$ & 2.130 & 0.539 & -0.539 & -0.206 & -0.070 & 0.070 & 0.065 & 0.013 & -0.013 & -0.083 & -0.004 & 0.004 \\
\hline 111 & -1.928 & -1.928 & -1.928 & 11.228 & 11.228 & 11.228 & 6.881 & 6.881 & 6.881 & -2.112 & -2.112 & -2.112 \\
\hline $31 \overline{1}$ & 2.115 & 0.679 & -0.679 & 1.590 & 0.416 & -0.416 & 1.945 & 0.211 & -0.211 & -1.120 & -0.548 & 0.548 \\
\hline 331 & -1.447 & -1.447 & -0.632 & 2.728 & 2.728 & 0.449 & 0.467 & 0.467 & 0.155 & -0.932 & -0.932 & -0.113 \\
\hline $35 \overline{1}$ & 0.249 & 0.533 & -0.032 & 0.399 & 0.702 & -0.132 & 0.167 & 0.557 & -0.086 & -0.229 & -0.476 & 0.127 \\
\hline 511 & 0.441 & -0.263 & -0.263 & 1.318 & 0.324 & 0.324 & 0.797 & 0.029 & 0.029 & -0.454 & -0.052 & -0.052 \\
\hline
\end{tabular}

interact with its eight NN by relaxed Coulomb interactions, and relaxed short-range Huggins-Mayer repulsive overlap forces including relaxed attractive dipole-dipole and dipole-quadrupole van der Waals interactions. Long-range Coulomb forces were considered in zero order for all host-lattice ions past the first shell.

A comparison of the Frenkel-pair formation energies given in Table I shows that for cation interstitials, the formation energy increases with increasing anion ionic radius, and that the opposite is true for anion interstitials. Also it is clearly evident that where the ratio of the ionic radii of anion to cation is significantly larger than unity, cation energies are lower than the corresponding anion case. Also, a comparison of $E_{F}^{+}$and $E_{F}^{-}$with the corresponding Schottky-pair formation energies $E_{s}$, shows that the latter has a lower formation energy in all cases. Thus, our results show that Schottky defect pairs are more likely to be present in crystals than are Frenkel defect pairs. ${ }^{6}$

In Ref. 1, we found that the increase in the Schottky-pair formation energy on going from approximation I to approximation II was largely due to the missing dipoles. Similarly, the addition of deformation dipoles on the anions will cause the Frenkel-pair formation energy to be higher. For example, if the distortion moment $P(r)$ is assumed to be proportional to the repulsive overlap so that $P(r)=P\left(r_{0}\right) e^{\left(r_{0}-r\right) / \rho}$, then the zero-order additional dipole contribution from the four NN anions about a cation interstitial will be nearly equal to the zero order missing dipole contribution, $2 \phi_{\mathrm{NN}}$, for all alkali halides. This contribution will cause the Frenkel-pair cation formation energies for the sodium family to be increased by about $0.5 \mathrm{eV}$ over the values given in Table I (the increase will be less for the remaining alkali halides). At present we do not know how to determine properly the zeroorder contribution for anion-anion deformation since it becomes difficult to make a good estimate of the distortion moment; however, we could argue that it is probably on the same order of magnitude as for cation-anion deformation. In either case the calculations clearly indicate that the Schottky pair should be the dominant type of defect.

A comparison of our Frenkel-pair formation energies for $\mathrm{NaCl}$ and $\mathrm{KCl}$ with other theoretical calculations is given in Table III. Since the models used are different, we can only say that they are of the same order of magnitude. We have also given the only seemingly available experimentally derived Frenkel-pair formation energy for a cation interstitial. This value for $E_{F}^{+}$was determined as a parameter in fitting experimental data for the ionic conductivity in $\mathrm{NaCl}$ and therefore the comparison should not be taken too seriously. However, the value does indicate an approximate range for $E_{F}^{+}$.

The displacements of ions about interstitials beyond NN are quite small when compared to the first neighbor displacements. This is similar to

TABLE III. Comparison of Frenkel-pair formation energies in $\mathrm{eV}$ for cations $(+)$ and anions $(-)$.

\begin{tabular}{|c|c|c|c|c|c|c|}
\hline Salt & $\begin{array}{l}\text { Theory } \\
\text { (present) }\end{array}$ & $\begin{array}{c}E_{F}^{+} \\
\text {Theory } \\
\text { (other) }\end{array}$ & Expt. & $\begin{array}{l}\text { Theory } \\
\text { (present) }\end{array}$ & $\begin{array}{c}E_{F}^{-} \\
\text {Theory } \\
\text { (other) }\end{array}$ & Expt. \\
\hline $\mathrm{NaCl}$ & 2.881 & $\begin{array}{l}1.36^{a} \\
2.63^{b} \\
2.9^{d}\end{array}$ & $2.699^{c}$ & 4.602 & $\begin{array}{l}4.60^{\mathrm{a}} \\
6.38^{\mathrm{b}}\end{array}$ & $\cdots$ \\
\hline $\mathrm{KCl}$ & 3.461 & $\begin{array}{l}2.56^{2} \\
3.42^{b}\end{array}$ & $\cdots$ & 3.734 & $\begin{array}{l}3.94^{2} \\
5.13^{b}\end{array}$ & $\cdots$ \\
\hline
\end{tabular}

${ }^{a}$ Calculation using a Born-Mayer repulsive potential by combining the values for $E_{s}$ from R. Guccione, M. P. Tosi, and M. Asdente [J. Phys. Chem. Solids 10, 162 (1959)] with $E_{I}$ from K. Tharmalingam [J。 Phys. Chem. Solids 25, 255 (1964)].

${ }^{b}$ Same as Ref. a above except a Born-Mayer-Verwey repulsive potential was used.

${ }^{c}$ Experimentally derived value from electrical conductivity measurements by A. R. Allnatt and P. Pantelis, Solid State Comm.6, 309 (1968).

${ }^{d}$ N. F. Mott and M. J. Littleton, Trans. Faraday Soc. 34, 485 (1938). 
the situation for vacancies. Also, the largest absolute displacement for these far ions is usually less than $3 \%$ of the equilibrium lattice spacing. Therefore, the correcting of defect host-lattice ion interactions past first neighbors for lattice relaxation will probably not alter the present values significantly, although again, making anharmonic corrections between first and second neighbors may prove to be worthwhile.

Another possible consideration for future study should be that of properly describing anion-anion deformation dipole interactions, and then allowing these additional dipoles (as well as those from cation-anion deformation) to interact with the elec- tric field at the NN lattice site. This field should include the contribution from the defect as well as the contributions from the host-lattice ions.

In conclusion, the method of lattice statics together with the deformation dipole model for ionic crystals has given a good description of cation and anion interstitial defects. Our results give confidence that an ion in any interstitial configuration can be properly treated. Thus, the methods and the manner of treating the defect host-lattice ion interactions for both the probiem considered here and the study of vacancies should prove to be useful for the study of other defect problems, e. g., vacancy pairs and vacancy migration configurations.
†Work performed in part under the auspices of the U. S. AEC at the University of California Lawrence Livermore Laboratory.

*Permanent address: Physics Dept., Abilene Christian College, Abilene, Tex. 79601.

${ }^{1}$ Paul D. Schulze and John R. Hardy, Phys. Rev. B 5, 3270 (1972).

${ }^{2}$ H. M. Evjen, Phys. Rev. 39,675 (1932).

${ }^{3}$ J. E. Mayer, J. Chem. Phys. 1, 270 (1933).

${ }^{4}$ F. G. Fumi and M. P. Tosi, J. Phys. Chem. Solids

\section{$\underline{25}, 31$ (1964).}

${ }^{5} \mathrm{~A}$ more complete listing of the ionic displacements and the dipole moments can be obtained from the authors upon request.

${ }^{6}$ At high temperatures Frenkel defects may become important in ionic conductivity for cases where $E_{F}$ is relatively low, e.g., $E_{F}^{+}$in $\mathrm{NaCl}, \mathrm{NaBr}, \mathrm{NaI}, \mathrm{KI}$, and $\mathrm{RbI} ; E_{F}^{-}$in $\mathrm{KF}$ and $\mathrm{RbF}$ where the cation has an ionic radius comparable to that of the anion. 